\title{
Guilleermo
}

de Delsham

Artículo original $\odot$ Acceso abierto

\section{Estado actual de la educación y la cultura hídrica: un mapeo sistemático de literatura}

\author{
Current state of water education and culture: \\ a systematic mapping of literature \\ Claritza Marlés Betancourt ${ }^{\mathrm{i}}$ (D) 0 \\ Lucelly Correa Cruz iD \\ i. Facultad de Ciencias Contables, Económicas y Administrativas. Universidad de la Amazonia; Florencias; Caquetá; Colombia.
}

Correspondencia. Claritza Marlés

Betancourt

Email: c.marles@udla.edu.co

Investigación: Artículo desarrollado en el marco del doctorado en Educación y

Cultura Ambiental.

Recibido: 29/02/2020

Revisado: 27/03/2021

Aceptado: 1/04/2021

Citar así: Marlés Betancourt, Claritza; Correa Cruz, Lucelly. (2021). Estado actual de la educación y la cultura hídricas: un mapeo sistemático de literatura. Revista Guillermo de Ockham. 19(1), 9-24. https://doi.org/10.21500/22563202.4591

Copyright: (C) 2021. Universidad de San Buenaventura, Cali.

La Revista Guillermo de Ockham proporciona acceso abierto a todo su contenido bajo los términos de la licencia Creative Commons AttributionNonCommercial-NoDerivatives 4.0 International (CC BY-NC-ND 4.0).

Conflicto de intereses. Los autores han declarado que no hay conflicto de intereses.

Disponibilidad de datos. Todos los datos relevantes están en el artículo. Para mayor información contactar al autor de correspondencia.

Fondos. Ninguno. Esta investigación no recibió ninguna subvención específica de agencias de financiamiento de los sectores público, comercial o sin fines de lucro.

Descargo de responsabilidad. El contenido de este artículo es responsabilidad exclusiva de los autores y no representa una opinión oficial de sus instituciones $\mathrm{n}$ i de la Revista Guillermo de Ockham.

\section{Resumen}

Los problemas relacionados con el medioambiente, en especial con el recurso hídrico, cada vez son más evidentes. A lo largo de los años la problemática ha avanzado sin tener un punto de retorno y a partir de esto se ha empezado a visualizar la educación como un aspecto primordial para el desarrollo de una cultura hídrica y generar seres humanos más consistentes y comprometidos con el entorno. El presente artículo indaga sobre el estado actual de las investigaciones acerca de la educación y la cultura hídricas, por lo cual contiene los resultados de un mapeo sistemático de literatura para identificar los estudios relacionados con la educación y cultura hídricas. En este sentido, se plantearon seis preguntas organizadas en dos dimensiones (cultural y educativa). El mapeo permitió seleccionar 50 estudios de diferentes fuentes que incluyen aportes acerca de las necesidades educativas y curriculares y el fomento y la finalidad de la cultura hídrica como elementos para la construcción de seres más conscientes y comprometidos con el recurso hídrico. Se identificó la necesidad de incluir prácticas interdisciplinarias y vincular componentes de educación ambiental e hídrica en instituciones y comunidades para privilegiar la participación y el compromiso social con el ambiente.

Palabras clave: educación, cultura, agua, hídrico, comunidad, política pública, educación ambiental.

\begin{abstract}
The problems related to the environment, especially to water resources, are increasingly evident; over the years the problem has advanced without having a point of return, from this point onwards education has begun to be seen as a fundamental aspect for the development of a culture towards water, generating more consistent and committed human beings with the environment. The purpose of this article is to investigate the current state of research on water education and culture; therefore, it contains the results of a systematic review and mapping of literature in relation to education and culture towards water. In this sense, 7 questions were posed, organized in two dimensions (cultural and educational). The mapping allowed the selection of 50 studies from different data sources, including contributions about educational and curricular needs, the promotion and purpose of water culture as elements
\end{abstract}


for the construction of beings more aware and committed to the water resource. The need to include interdisciplinary practices and link environmental and water education components in institutions and communities was identified in order to give priority to participation and social commitment to the environment.

Keywords: education, culture, water, water, community, public policy, environment education.

\section{Introducción}

En la actualidad, es alto el reconocimiento de los comportamientos antrópicos y sus efectos frente a las problemáticas ambientales e hídricas. En tal sentido, lo hídrico se considera un elemento vital por su impacto determinante en las dinámicas socioambientales de carácter intergeneracional. En este aspecto, es necesario enfatizar acerca de la necesidad de cambiar las actitudes y acciones en la relación entre el hombre y la naturaleza.

Acorde con lo expuesto, los procesos educativos y culturales son considerados el cimiento para la consecución de cambios comunitarios que promuevan actitudes y acciones proambientales. Estos procesos son relevantes porque inciden en la formación de personas sensibles, conscientes de su impacto en la naturaleza y con capacidades necesarias para abordar dichas problemáticas.

En esta perspectiva, se concibe que la educación y la cultura hídricas son aspectos relevantes en la identificación e implementación de alternativas para afrontar la problemática ambiental, en la medida en que se puede incidir en el proceso de sensibilización y concientización para el cuidado del entorno natural (Correa, López, y Vergara, 2020); razón por la cual es factible proponer acciones orientadas a mitigar el detrimento del entorno natural.

Desde la perspectiva educativa, el abordaje hídrico es relevante, pues posibilita la presentación de propuestas relacionadas con estrategias pedagógicas, didácticas y curriculares. De ahí que la educación represente una alternativa para influir en los procesos de transformación cultural y direccionarlos hacia la formación integral de seres humanos reflexivos, conscientes y proactivos en torno a la realidad ambiental.

La educación hídrica o del agua requiere ser interpretada desde un enfoque multidisciplinario, toda vez que involucra el trabajo conjunto de profesionales, la comunidad y los medios de comunicación, entre otros protagonistas que pueden aportar a la comunicación de problemas del agua de manera sencilla y asequible (Unesco, 2018). Para ello, es necesario que las prácticas de enseñanza estén fundamentadas "bajo el concepto de que aprender no sea solo conocer, sino también reconocer cómo hacer frente a los problemas relativos al agua" (Unesco, 2020a, p. 51).

$\mathrm{Al}$ respecto, se considera que la cultura hídrica o del agua está relacionada con las costumbres, las actitudes y los valores compartidos en la dupla individuo-sociedad, en relación con la comprensión de lo hídrico; es decir, de la importancia del agua en lo coherente con la vida (Cárdenas, 2018). En tal sentido, es imperante propender por comportamientos y acciones orientadas al cuidado, renaturalización y preservación de fuentes hídricas; es decir, "hacia la concientización del impacto de comportamientos humanos, en el entorno natural” (Correa, 2020, p. 131).

En esta perspectiva, el presente artículo contiene los resultados de un mapeo sistemático que permite obtener información valiosa sobre el estado actual de las investigaciones acerca de la educación y la cultura hídricas. Mediante un análisis documental, se identificaron y categorizaron estudios e investigaciones publicadas en diferentes bases de 
datos. Al respecto, se indagaron las siguientes dimensiones de análisis: 1. la dimensión investigativa, y 2. la dimensión temática. En la dimensión investigativa, se abordaron los siguientes interrogantes: ¿̨cuáles son los estudios sobre educación y cultura hídrica en publicaciones científicas?, ¿cómo están distribuidos geográficamente los estudios sobre educación y cultura hídrica?, y ¿qué tipo de estudios se han realizado relacionados con educación y cultura hídrica? En lo concerniente a la dimensión temática, se indagó sobre: ¿cuáles son los factores temáticos identificados en relación con la educación y cultura hídrica frente a la crisis hídrica?, ¿cuál es el tipo de población en la cual se desarrollan los procesos de educación y cultura hídrica?, y ¿cuántos estudios han presentado resultados relacionados con la articulación de la educación y cultura como alternativas para afrontar la problemática hídrica?

En lo concerniente a la temática de estudio denominada educación hídrica o educación del agua, los hallazgos obtenidos se relacionan con las subcategorías enseñanza y aprendizaje sobre el agua, procesos curriculares, y desafíos de la educación hídrica. Los aspectos convergentes están asociados con la configuración de aspectos educativos y comportamientos sociales en el impacto ambiental e hídrico.

Acerca de la temática de estudio sobre cultura hídrica $(\mathrm{CH})$, las subcategorías identificadas son las problemáticas y alternativas de solución, la divulgación de conocimientos hídricos y la apropiación de la cultura hídrica. En los documentos indagados en esta temática de estudio, se encontró el accionar del ser humano como aspecto prioritario en la valoración y en el impacto hídricos.

En coherencia con lo planteado, se ha trazado el siguiente objetivo de investigación: indagar sobre el estado actual de las investigaciones acerca de la educación y la cultura hídricas, razón por la cual se enfatiza en los resultados arrojados en el mapeo sistemático. De este modo, se incluyó el análisis de los aportes identificados en los documentos consultados respecto a cada una de las subcategorías ya mencionadas. De manera similar, se presentan los interrogantes planteados en este estudio, los cuales se establecieron en torno a dimensiones, bases de datos en las cuales se hallaron los documentos, ubicación geográfica, factores temáticos, estrategias de búsqueda y selección de investigaciones. En consecuencia, se plantean la discusión y conclusiones.

\section{Metodología}

El desarrollo de la investigación se llevó a cabo mediante mapeo sistemático, un método útil para construir la clasificación de los documentos consultados, obtener la información sobre planteamientos e identificar vacíos y posibles comparaciones entre el tema de estudio y el material consultado (Jácome, Ordóñez, Cerón y Villaquirán, 2018). Según Velásquez (2014), en los documentos hallados se analizan resultados y aspectos relevantes de la información sobre la temática de estudio, encontrada en diferentes bases de datos. Acorde con lo expuesto, se presenta la siguiente matriz analítica (Tabla 1):

Mediante esta matriz analítica se presentan los hallazgos encontrados y analizados en torno a la educación y la cultura hídricas. Lo expuesto en la matriz es el consolidado de los hallazgos relevantes obtenidos mediante las fases exploratoria, concerniente a la búsqueda y selección de documentos, y la fase interpretativa, mediante la cual se procedió con el proceso de revisión y análisis. En ella se encontraron las siguientes categorías emergentes: aprendizaje, enseñanza, currículo, desafíos educativos, divulgación del conocimiento sobre lo hídrico, problemas y alternativas de solución y apropiación de la cultura hídrica.

Aplicar este método de análisis posibilitó una clasificación de los temas de estudio mediante el enfoque y los resultados de las investigaciones, a partir del estudio de 50 do- 
Tabla 1

Matriz analítica. Educación y cultura hídricas

\begin{tabular}{|c|c|c|c|}
\hline \multicolumn{2}{|r|}{ Educación hídrica } & \multicolumn{2}{|r|}{ Cultura hídrica } \\
\hline Resultados & Aspectos relevantes & Resultado & Aspectos relevantes \\
\hline Aprendizaje & $\begin{array}{l}\text { Educación holística, omnicomprensiva. } \\
\text { Textos, fenómenos, lúdicas. } \\
\text { Procesos participativos y pedagógicos. }\end{array}$ & $\begin{array}{l}\text { Divulgación del conoci- } \\
\text { miento sobre lo hídrico. }\end{array}$ & $\begin{array}{l}\text { Inclusión de campañas y publicidad sobre el } \\
\text { agua. } \\
\text { Participación comunitaria. } \\
\text { Investigación e implementación de proyectos. } \\
\text { Inclusión de espacios educativos y culturales. }\end{array}$ \\
\hline Enseñanza & $\begin{array}{l}\text { El papel del maestro en el fomento de las prácticas } \\
\text { educativas. } \\
\text { Propender por la comprensión de la realidad. } \\
\text { Promover hábitos y rutinas que lleven a la apropia- } \\
\text { ción de lo hídrico. }\end{array}$ & $\begin{array}{l}\text { Problemas y alternativas } \\
\text { de solución. }\end{array}$ & $\begin{array}{l}\text { Reconocimiento de cosmovisiones ancestrales. } \\
\text { Conocimiento del territorio. } \\
\text { Formación ciudadana con sentido de perte- } \\
\text { nencia. }\end{array}$ \\
\hline Currículo & $\begin{array}{l}\text { Lo hídrico está casi ausente en los procesos de } \\
\text { formación. } \\
\text { Existe carencia del trabajo interdisciplinario. } \\
\text { En los planes de estudio se establece qué enseñar, } \\
\text { pero no se orienta cómo. }\end{array}$ & \multirow{2}{*}{$\begin{array}{l}\text { Apropiación de la cultu- } \\
\text { ra hídrica. }\end{array}$} & \multirow{2}{*}{$\begin{array}{l}\text { Valoración de lo hídrico. } \\
\text { Vinculación en procesos de política pública. } \\
\text { Implementación de programas y proyectos. } \\
\text { Conocimiento, actualización e implementa- } \\
\text { ción de regulaciones normativas. }\end{array}$} \\
\hline Desafios educativos. & $\begin{array}{l}\text { Falta mayor exploración de enfoques pedagógicos } \\
\text { que promuevan el pensamiento crítico. } \\
\text { Falta mayor participación ciudadana. } \\
\text { Gran parte de la información hídrica es muy } \\
\text { técnica. }\end{array}$ & & \\
\hline
\end{tabular}

cumentos hallados en diferentes bases de datos, con base en el año publicado, la temática, las subcategorías identificadas, los objetivos, los resultados y las fuentes de consulta, para obtener una visión general del tema de estudio.

\section{Planificación del mapeo y definición de interrogantes de investigación}

Para llevar a cabo el mapeo sistemático, se definieron preguntas investigativas de acuerdo con dos dimensiones de análisis: la dimensión investigativa y la dimensión temática.

\section{Dimensión investigativa}

Se presentan los interrogantes planteados en relación con las características de la información consultada. Esta dimensión hace referencia al material investigativo consultado y abarca cuestiones sobre los estudios existentes en las temáticas de educación y cultura hídricas, la distribución geográfica de estas publicaciones y el tipo de estudio llevado a cabo. Los interrogantes planteados fueron los siguientes:

1. ¿Cuáles son los estudios sobre educación y cultura hídricas en publicaciones científicas?

2. ¿Cómo están distribuidos geográficamente los estudios sobre educación y cultura hídricas?

3. ¿Qué tipo de estudios se han llevado a cabo relacionados con la educación y la cultura hídricas?

La especificación de los resultados concernientes a la dimensión investigativa posibilita la ejecución de las investigaciones publicadas en el campo de la educación y la cultura hídricas, a partir de las cuales se fundamentan los resultados del mapeo. En este sentido, se facilita el reconocimiento del país de origen de los aportes hechos por la comunidad académica y científica. Adicionalmente, permite el reconocimiento de los principales tipos de publicación sobre el problema, mediante la exploración de propuestas teóricas 
y empíricas, con base en el hecho de que la investigación teórica compara ideas entre sí, y la investigación empírica compara las ideas con la realidad (Cazau, 2006, p. 32).

\section{Dimensión temática}

Se exponen los planteamientos presentados en investigaciones sobre la temática indagada y el tipo de población participante. En esta perspectiva, los interrogantes formulados fueron:

4. ¿Cuáles son los factores temáticos identificados en relación con la educación y la cultura hídricas frente a la crisis hídrica?

5. ¿Cuál es el tipo de población en la cual se desarrollan los procesos de la educación y la cultura hídricas?

6. ¿Cuántos estudios han presentado resultados relacionados con la articulación de la educación y cultura como alternativas para afrontar la problemática hídrica?

Los hallazgos concernientes a la dimensión temática posibilitan la identificación del problema en el que se desarrolla la educación y la cultura hídricas, mediante la determinación de escenarios e instituciones educativas participantes en los estudios encontrados y la articulación de lo cultural y lo ambiental respecto a lo hídrico.

\section{Resultados}

En el presente apartado se exponen en detalle los aspectos concernientes a la implementación del mapeo y se presentan los hallazgos identificados a cada uno de los interrogantes presentados en relación con la dimensión investigativa y la dimensión temática.

\section{Dimensión investigativa}

Las temáticas abordadas fueron: 1. estudios sobre educación y cultura hídricas; 2. distribución geográfica de los estudios indagados, y 3. tipos de estudios sobre el tema investigado. Por consiguiente, a continuación se presenta lo encontrado en cada uno de los aspectos mencionados. Con esto se puede plantear una matriz para encontrar los matices más relevantes a nivel investigativo con el recurso hídrico, para poder aportar en la generación de nuevo conocimiento.

\section{Estudios sobre la educación y la cultura hídricas}

Las investigaciones consultadas fueron encontradas en bases de datos como Dialnet, Onlinelibrary, Redalyc, Scielo y Elsevier. Al respecto, se identificaron en primer lugar, las investigaciones sobre educación, en las cuales predominan las temáticas de enseñanza y aprendizaje, seguidas de procesos curriculares y desafíos de la educación, y en segundo lugar, lo relacionado con la temática de cultura del agua. Los documentos referenciados en las subcategorías de análisis se basaron en temáticas ligadas a problemáticas del recurso y alternativas de solución. Una cuestión menos abordada se basa en la apropiación de una cultura hídrica en comunidades académicas y sociales.

\section{Distribución geográfica de los estudios sobre educación y cultura del agua}

Se hizo un reconocimiento de los países de origen respecto de la temática indagada. Los países en los cuales los investigadores han presentado mayor interés temático, se pueden identificar en la Figura 1. 
Figura 1

Países de origen por temáticas indagadas

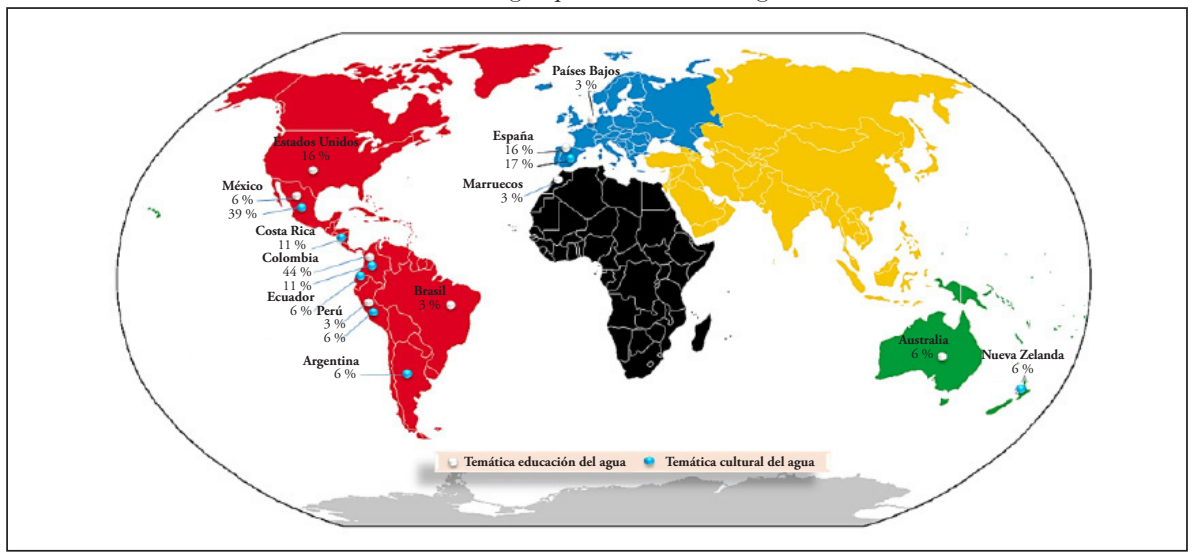

Acerca de la educación hídrica, el país con mayor número de investigaciones sobre temáticas concernientes a procesos de enseñanza y aprendizajes es Colombia. Los asuntos menos indagados fueron los relacionados con procesos curriculares (Australia y Marruecos) y desafíos de educación (Brasil, Países Bajos, México y España). Acorde con lo identificado, se puede inferir que en Colombia este fenómeno responde a la inclusión impuesta de la educación ambiental, a causa de políticas educativas y reglamentos que conllevan una planificación con currículos que contienen cursos obligatorios, sin el previo reconocimiento de los diversos contextos. Por lo tanto, se considera que "la educación ambiental en Colombia requiere de un modelo pedagógico bien estructurado, que integre procesos de enseńanza decisivos, enmarcados hacia el medioambiente, con el compromiso primordial de todos los individuos" (Carrillo y Cacua, 2019, p. 1).

En lo concerniente a la temática de cultura hídrica, la subcategoría en donde se halló un mayor número de documentos fue la relacionada con problemáticas y alternativas de solución (México, Nueva Zelanda y España). En estos países, la cultura hídrica adquiere relevancia por su impacto en los modelos de producción agraria, la seguridad alimentaria y los procesos de saneamiento (Pérez, 2015, p. 26). Al respecto, se destaca que el interés investigativo sobre la apropiación de la cultura hídrica está relacionado con problemas como la escasez del agua y la crisis alimentaria.

\section{Tipos de estudios abordados en las investigaciones consultadas}

En los estudios consultados sobre la temática educación y cultura hídrica, se han desarrollado indagaciones relacionadas con los aspectos teóricos y empíricos (Gráfico 1).

\section{Gráfico 1}

Tipos de investigación llevadas a cabo en documentos relacionados con las temáticas de estudio

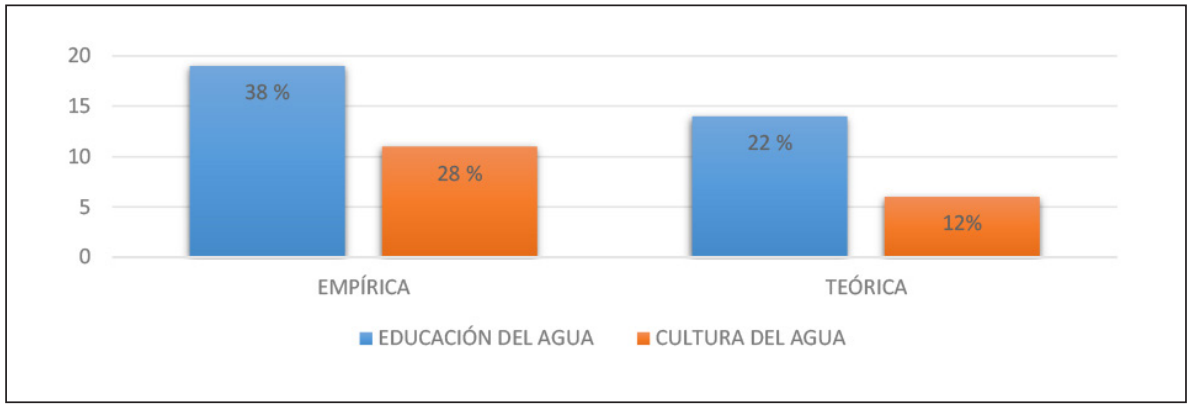


Según lo identificado, se reconoce que en los estudios sobre educación hídrica y cultura hídrica prevalecen un enfoque empírico y los trabajos con diversas comunidades académicas y sociales que aportan información significativa sobre las causas y consecuencias de las problemáticas indagadas sobre el recurso hídrico. No obstante, dada la importancia de los estudios cualitativos es necesario abordar nuevas investigaciones desde enfoques cuantitativos y plurimetodológicos que pueden ser medibles y verificables.

\section{Factores temáticos identificados}

En este apartado, se exponen los hallazgos concernientes a la educación y la cultura hídricas.

\section{La educación hidrica}

En este campo se relacionaron documentos de investigación con énfasis en tres subcategorías de análisis: enseñanza y aprendizaje, procesos curriculares y desafíos de la educación.

Respecto de la enseñanza y aprendizaje, los procesos educativos se configuran como una alternativa para mejorar actitudes y comportamientos sociales frente al entorno natural, pues se pueden fomentar procesos de reflexión y valores proambientales (Monja, 2018). Al respecto, Freitas y Marín (2015), señalan al maestro como orientador de prácticas educativas para el reconocimiento del contexto y la realidad locales. En esta perspectiva, se propende por la reconstrucción de la relación del hombre con el ambiente, mediante procesos de lectura, comprensión de textos y sensibilización ambiental (Campos y García, 2017).

El fomento de procesos de formación es importante dada la vinculación de la comunidad en la toma de decisiones educativas. Entre las alternativas planteadas se destacaron los siguientes aspectos: a. proyectos de educación ambiental; b. educación formal y no formal; c. espacios de sensibilización; d. apropiación de TIC, y e. gestión hídrica (Tabla 2).

El proceso de enseñanza y aprendizaje implica la identificación de actitudes y comportamientos sociales (Fernández y Rodríguez, 2016). Al respecto, se destacan como desafíos la configuración de un pensamiento crítico, la concienciación y la comprensión de la naturaleza y el impacto de la huella hídrica desde la transformación de estilos de vida y el fomento de la responsabilidad social (Missingham, 2013; Mahler, 2019).

En cuanto a los procesos curriculares, se destacó el énfasis en procesos interdisciplinarios, con dimensión pedagógica para la transformación económica y social a partir de

Tabla 2

Alternativas plateadas en los procesos de formación sobre el agua

\begin{tabular}{lll}
\hline \multicolumn{1}{c}{ Alternativa } & \multicolumn{1}{c}{ Argumentos } & \multicolumn{1}{c}{ Autores } \\
\hline Proyectos y programas & $\begin{array}{l}\text { Se requieren programas de educación ambiental para aportar a } \\
\text { la gestión hídrica. }\end{array}$ & Cárdenas, Reyes y Bustos, 2017. \\
\hline Educación formal y no formal & $\begin{array}{l}\text { Es fundamental desde el hogar, para el fomento de actitudes } \\
\text { proambientales. }\end{array}$ & $\begin{array}{l}\text { Venckute, Moreira y Figueiredo, 2017; Sanz, } \\
2018 .\end{array}$ \\
\hline Espacios de sensibilización & $\begin{array}{l}\text { Permiten la configuración de una conciencia ambiental. Los } \\
\text { talleres y artes plásticas permiten rescatar el valor del agua como } \\
\text { fuente de vida. }\end{array}$ & $\begin{array}{l}\text { Sanz, 2018, Carrillo, Carrillo y Pena, 2017; } \\
\text { Gil, Quílez, Peña, Hervás y Muñoz, 2018. }\end{array}$ \\
\hline Apropiación de TIC & $\begin{array}{l}\text { Implica la inclusión de estrategias educativas mediante procesos } \\
\text { tecnológicos innovadores y motivadores; entre las alternativas se }\end{array}$ & $\begin{array}{l}\text { Moreno, Vahos y Mazo, 2019; Angarita, } \\
\text { Duarte, y Fernández, 2018; Galván, Ouaria- } \\
\text { chi, Pozo y Gutiérrez, 2018. }\end{array}$ \\
\hline Gestión hídrica comunitaria & Se requiere liderazgo de instituciones educativas para la gestión & $\begin{array}{l}\text { Grigg, 2019; Hernández y Gómez, 2014; } \\
\text { del agua con incidencia en los planes de ordenamiento. }\end{array}$ \\
\hline
\end{tabular}


prácticas, discusiones y métodos (Jardioui, Elachqar, Kaddaria y Ijjaalib, 2015; Sierra, Medina y Aguilera, 2018).

Acorde con lo indagado, Sammel (2016) sugiere que la educación formal (ofertada por instituciones) y la no formal (se produce en grupos y organizaciones en comunidad), debe estar enfocada en la formación sobre el ambiente, al reconocer que el recurso hídrico es un eslabón y las diferentes problemáticas que enfrenta afectan las comunidades desde los ámbitos económico, social y ambiental. Para la Unesco (2020b, p. 191), se requiere voluntad política y liderazgo para incorporar el valor del agua en los acuerdos y tratados mundiales, al existir una desconexión entre las acciones de gestión hídrica y la política internacional.

El análisis de construcción de currículo para el agua se centra en la formación ciudadana mediante la concientización ambiental colectiva. Por ello, los planes de estudio requieren priorizar la visión del entorno natural y el papel de individuo en este, vincular acciones curriculares a partir del conocimiento de los problemas locales, identificar problemáticas, usos, actitudes y responsabilidades individuales con el ambiente (Marlés, Rojas y Correa, 2020, p. 215). Es prioritario que la educación para el agua incorpore la transdisciplinariedad y la perspectiva de las problemáticas ecológicas, socioeconómicas y políticas a partir de la glocalidad.

Respecto de los desafíos en la educación, en la compresión de problemática hídrica y el papel de ser humano en el sistema natural, los investigadores plantearon como desafíos educativos la formación integral, la academia, la educación no formal y la participación comunitaria. Esta última exige conjugar el conocimiento local y natural con la técnica que ofrece la academia (Hernández \& Palacios, 2016) (Tabla 3).

Según lo planteado, el reto educativo está configurado en procesos de investigaciónacción con la inclusión de la comunidad, para lograr trabajos mancomunados orientados hacia la solución de problemas ambientales (Delgado, Trujillo \& Torres, 2013). Es necesario aclarar que las diversas comunidades enfrentan una crisis hídrica mundial, resultado de problemas de gestión hídrica, malos hábitos y la limitada normatividad para regular el consumo, problemáticas que exigen la formulación de alternativas para la preservación, el uso sustentable del recurso hídrico y la participación social.

\section{Cultura hídrica}

Esta es la segunda temática abordada en los estudios consultados. Los aspectos que se destacaron son: a. divulgación de conocimiento; b. problemáticas y alternativas de solución, y c. apropiación de la cultura hídrica.

Tabla 3

Desafíos en los procesos educativos

\begin{tabular}{lll}
\hline \multicolumn{1}{c}{ Alternativa } & \multicolumn{1}{c}{ Argumentos } & \multicolumn{1}{c}{ Autores } \\
\hline Formación integral & $\begin{array}{l}\text { El desarrollo de habilidades, actitudes y destrezas } \\
\text { necesarias permite afrontar la realidad natural del } \\
\text { siglo XXI por medio del aprendizaje inter y multi } \\
\text { disciplinario. }\end{array}$ & $\begin{array}{l}\text { Kulcsar, Aistrup, Bulatewicz, Peterson, Welch, y Steward, } \\
\text { 2016; Bolaños, 2017; Read y Garcia, 2015. }\end{array}$ \\
\hline $\begin{array}{lll}\text { Educación no formal y participa- } \\
\text { ción comunitaria }\end{array}$ & $\begin{array}{l}\text { Es un espacio de aprendizaje, mediado por la } \\
\text { experiencia y la observación. Es importante la vin- }\end{array}$ & Cada, y Alvis, 2016; Oyaga, Calderon, Olaya, Enamorado comunitaria para facilitar la apropiación \\
& del conocimiento local. & y Atencio, 2017; Díez, 2014 \\
\hline \multirow{2}{*}{$\begin{array}{l}\text { Academia y ámbito laboral } \\
\text { El reto de la universidad está en vincular estudios }\end{array}$} & Haynes, y Cech, 2015 \\
& $\begin{array}{l}\text { del agua para todas las carreras académicas } \\
\text { ofertadas. }\end{array}$ & \\
\hline
\end{tabular}


En cuanto a la divulgación del conocimiento sobre lo hídrico, el agua, como fuente de vida, ha cobrado cada vez más importancia en diferentes espacios del desarrollo de la comunidad y su entorno. Sin embargo, los esfuerzos son llevados a cabo por cierto número de personas y no por una comunidad en general. Entre las alternativas para la divulgación de conocimientos sobre lo hídrico, Ortega y Peña (2016) plantearon campañas promocionales para influenciar los patrones socioculturales de los hogares, pero estas alternativas dejan a un lado sectores industriales, agropecuarios y organizacionales, a lo que se suma que estas actividades no provienen de macroproyectos con visión integral y participativa de la comunidad como protagonista principal en la apropiación y promoción de experiencias vinculadas al desarrollo de una cultura hídrica (Carrillo, Carrillo \& Pena, 2017).

En las propuestas para generar una cultura hídrica, se reconoce la creación de espacios educativos y culturales mediante la aplicación de material lúdicos y académicos para el fortalecimiento de la cultura hídrica y la concientización ciudadana (López, 2017).

En lo relativo a las problemáticas y alternativas de solución, se consideró necesario iniciar con el estudio de las cuestiones hídricas (almacenamiento, distribución, repartición, aprovechamiento, uso y consumo), para crear una cultura hídrica a partir de los aportes de las comunidades. El concepto de agua, comprendido según la visión económica, evidencia la importancia de concebir el agua como vida y esencia de la cultura del ser humano. En esta perspectiva, el aporte de las cosmovisiones ancestrales permite ver mejor, a través del "lente espiritual", la relación del agua y el pueblo (González y Vega, 2018).

En este aspecto, se destaca el papel del Gobierno para fomentar el estudio de las problemáticas hídricas de cada contexto (Macpherson y Macpherson, 2017). En tal sentido, se identificó que el fortalecimiento de una cultura hídrica o cultura del agua permitirá la formación de ciudadanos con sentido de pertenencia. Por ende, hablar de una nueva cultura del agua no solo hace énfasis en temáticas éticas, sino que se crea un debate ambiental y económico en la gestión y gobernanza hídricas (Pérez, 2015; Fuentes, 2012).

Sobre la apropiación de la cultura hídrica, la poca valoración de lo hídrico se evidencia en la sobreexplotación y los comportamientos antrópicos. En esta óptica, para la construcción de una cultura hídrica es necesario contar con conocimientos ambientales e hídricos, espacios para pensar, vivir y sentir el entorno de manera diferente, una nueva escala de valores aunados a estilos de vida fundamentados en procesos de responsabilidad ciudadana, vinculación en procesos de política pública e implementación de programas y proyectos de orden nacional y local enfocados en el recurso hídrico (González y Arzaluz, 2011; Topete, Verdín y Galaviz, 2019).

Según lo expuesto, se tiene un problema del recurso hídrico que va en aumento y se manifiesta en escasez, contaminación y pérdida de ecosistemas hídricos, como resultado de factores antrópicos. Ello evidencia la necesidad de instaurar una cultura hídrica para consolidar los valores de ahorro, conservación y utilización eficiente del agua (Ortega y Peña, 2016). Así mismo, lo hídrico se reconoce por su relevancia social en el fomento de una cultura del agua (Castelltor, 2015). Para ello, se proponen campańas sobre el uso racional de este elemento, procesos de formación ciudadana, concentración de esfuerzos en planes y proyectos ambientales e hídricos, que requieren ser analizados con base en aspectos culturales, ecológicas y políticos, a fin de reconocer el impacto de la interrelación entre el hombre y la naturaleza (Koberwein, 2017; Damonte y Lynch, 2016).

En consecuencia, la configuración de la cultura hídrica es importante toda vez que los procesos de sensibilización y concientización tienen incidencia en la mitigación de los impactos ambientales (Figueroa, 2017). Al respecto, Ortega (2015) reconoce la necesidad de ver al agua no solo como un bien, sino también como un aspecto fundamental 
del desarrollo del individuo y la sociedad. En este proceso se denota la identificación de habilidades, creencias, actitudes y valores de la comunidad (Moreira, Araya y Charpentier, 2015).

Otro aspecto en la construcción de una cultura hídrica, de acuerdo con Verdín, Topete, Galaviz, Ponce y Soriano (2016), comprende la relación del conocimiento acerca del agua y la interrelación de su legislación. En este sentido, las normas jurídicas deben estar en relación con las nuevas necesidades de las comunidades. De ahí la importancia de las investigaciones sobre el agua y su cultura en relación con el planteamiento e implementación de propuestas en favor del cambio de comportamientos antrópicos (Mazabel y Fragoso, 2012).

En la actualidad, las diferentes políticas educativas y ambientales han sido afectadas por la crisis económica y los recortes presupuestales, lo que lleva a procesos de educación ambiental que muchas veces se quedan en el discurso y en la aplicación fragmentada de currículos impuestos. Adicionalmente, algunos aspectos normativos se han quedado obsoletos y tienen poca aplicabilidad en contextos específicos. Tal precarización en la disponibilidad hídrica está relacionada de manera directa con las formas dominantes de apropiación del agua y las diferentes actividades de exploración minera y energética, exportación agrícola y plantaciones forestales, como actividades características de los modelos que generan desarrollo mediante el despojo de recursos naturales en los territorios (Castelltor, 2015; Panéz, 2017).

\section{Tipo de población en la cual se desarrollan los procesos de educación y cultura hídrica}

Acerca de la educación hídrica, la población investigada se focalizó en participantes del ámbito educativo y comunitario. En este sentido, en las investigaciones se trabajó con estudiantes de diferentes niveles de educación, desde primaria, secundaria hasta universitario. De igual manera, se hicieron estudios con comunidades afectadas por la crisis hídrica.

En las indagaciones que abordaron el ámbito educativo, se identificó la necesidad de incluir prácticas interdisciplinarias y vincular componentes de educación ambiental e hídrica. En el análisis de las comunidades, se encontró como requerimiento la apropiación de una educación ambiental mediante el desarrollo de prácticas académicas lideradas por centros educativos y la apropiación de actividades culturales, artísticas y programas computacionales que propicien conocimientos contextuales sobre lo hídrico.

En lo referente a la temática cultura hídrica, se encontró que para la mitigación de los impactos ambientales e hídricos es relevante priorizar el involucramiento de las comunidades y la determinación del impacto de las campañas publicitarias sobre lo ambiental. Por otra parte, se indagó sobre la formación de cultura hídrica en pobladores de las microcuencas, para hacer una toma de conciencia acerca de las problemáticas que generan las acciones humanas sobre estos recursos. Por último, se analizó lo concerniente a la aplicación de políticas de mitigación de impactos hídricos y la necesidad de formar una cultura hídrica con las comunidades.

Además, Moreira, Araya \& Charpentier (2015), exponen que la identificación de conocimientos, habilidades, creencias y valores de las personas es indispensable para la construcción de una cultura hídrica, por ser los directos involucrados en el proceso y los resultados obtenidos, sobre la base de que las respuestas a problemas reales afectan las necesidades e intereses de las comunidades, convirtiendo así la educación en la clave para instaurar cambios en las prácticas, en la creación de actitudes y aprendizajes. 
Estudios que han presentado resultados relacionados con la articulación de la educación y cultura como alternativas para afrontar la problemática hídrica

Acerca de la articulación entre la educación y la cultura hídrica, los aspectos presentados en las investigaciones consultadas se exponen en la Tabla 4.

Los procesos educativos son considerados elementos fundamentales en la configuración de una cultura hídrica, pues la educación "forma parte de los elementos simbólicos de la cultura; por ende, es parte de la problemática ambiental” (Bonilla y Vera, 2011, p. 179). En esta perspectiva, el reconocimiento de la problemática ambiental e hídrica de cada territorio puede contribuir a la configuración de una conciencia ambiental (Miranda, 2013; Correa, Pascuas y Marlés, 2016; Lara y Otaño, 2017).

De acuerdo con la Unesco (2020b), la problemática actual de los recursos hídricos evidencia la necesidad de reconocer, cuantificar y expresar el valor de este recurso en sus diferentes usos, como síntoma principal de la desatención política hacia el agua y los problemas de gestión, que en su gran mayoría no considera el agua un factor relevante para la toma de decisiones. En este sentido, la educación es fundamental para fomentar una cultura hídrica que contribuya a la valoración del agua, de manera específica la educación ambiental; concebida como la base fundamental para la transformación de comportamientos antrópicos, mediante procesos participativos que articulen conocimientos que incidan en procesos de política pública ambiental, en aras de mitigar el detrimento hídrico (Severiche, Gómez y Morales, 2016).

\section{Discusión y conclusiones}

Las diferentes problemáticas que se asumen en relación con el recurso hídrico en diferentes regiones del mundo requieren la inclusión de procesos formativos orientados a la consolidación de una cultura hídrica. Al respecto, se considera que más allá de hacer campañas para generar consciencia en las comunidades, se debe tratar la educación como pilar en la formación ciudadana y en la transformación de comportamientos sociales frente a los recursos hídricos.

En el análisis documental llevado a cabo, acorde con las tendencias investigativas sobre la educación y la cultura hídricas, se establece la preocupación de los autores por generar alternativas frente al uso desmesurado que se les da a los recursos hídricos. De manera consciente, se discute el papel del ser humano en el cambio de esta dinámica altamente extractiva y contaminante y se reconoce que ya es momento de actuar como comunidad para lograr cambios en la práctica social frente a los recursos hídricos, iniciando por la educación a temprana a edad.

Tabla 4

Investigaciones sobre educación y cultura hídrica

\begin{tabular}{|c|c|}
\hline Aspectos relevantes & Autor (año) \\
\hline $\begin{array}{l}\text { Fomentar acciones para generar un espacio de cultura del agua, mediante eventos de difusión del conocimiento, en } \\
\text { actividades educativas, académicas y culturales, apropiación de actividades lúdicas, talleres o cursos para capacitar a las } \\
\text { comunidades sobre la construcción y fortalecimiento de capacidades en fomento de una cultura del agua. }\end{array}$ & $\begin{array}{l}\text { Ortega y Peña (2016); } \\
\text { Marlés, Peña y Gómez } \\
(2017)\end{array}$ \\
\hline $\begin{array}{l}\text { El plan de desarrollo sobre educación ambiental debe contribuir a las competencias necesarias para lograr la ejecución de } \\
\text { acciones ambientales, y permitir que el individuo tome acciones individuales como primer mecanismo en la búsqueda de } \\
\text { integrar acciones colectivas y comunales, con el fin de lograr el aseguramiento del recurso hídrico. }\end{array}$ & $\begin{array}{l}\text { Moreira, Araya \& Char- } \\
\text { pentier (2015) }\end{array}$ \\
\hline $\begin{array}{l}\text { Los fundamentos educativos para la construcción de una cultura del agua, permiten formar niños y jóvenes replicadores } \\
\text { de información, para la construcción de ciudadanos más conscientes y construidos acerca de afrontar la crisis hídrica y el } \\
\text { cuidado del agua. }\end{array}$ & López (2017) \\
\hline
\end{tabular}

Establece el surgimiento de la cultura ambiental como elemento de la educación ambiental que el hombre recibe en su crecimiento personal, como resultado de la transformación de su conciencia social e individual, así como de sus valores, sentimiento y actitudes, dicha construcción permitirá seres más conscientes acerca de su entorno y sus problemáticas. 
La educación hídrica debe asumir el reto de reformular los currículos de formación en los diferentes niveles de educación. Debe ser responsabilidad de todas las áreas de formación incluir los aspectos ambientales de manera transversal y así el individuo comprendería cómo su actuar genera impacto en el entorno y de qué manera puede contribuir en la generación de cambios en esa dinámica, a partir de un contexto individual, con proyección local y global.

Es un desafío tanto para las comunidades como para la educación, lograr un cambio en la manera como los individuos y las diferentes instituciones, empresas e industrias dan uso a sus recursos hídricos y de qué manera logran mitigar los daños causados. En tal sentido, es necesario trascender la concepción neoliberal, pues el agua no puede ser asumida como mercancía relacionada con factores agroecológicos, energéticos, alimentarios o socioeconómicos.

Así mismo, es necesario incluir aspectos relevantes que forman parte de la educación y el desarrollo en el campo del recurso hídrico, así como la afectación que diversos sectores económicos hacen sobre el recurso hídrico como, por ejemplo, la minería y el sector agroindustrial, los cuales generan casi un $70 \%$ de la huella hídrica y que por ningún lado, es abordado en la investigación, pero que tácitamente se sabe que existen.

En esta perspectiva, para lograr una cultura y conciencia hídricas, se considera que la educación ambiental e hídrica es un elemento fundamental para promover comportamientos proambientales. No obstante, se requiere la reestructuración de los currículos de formación, la inclusión de procesos de formación ciudadana, la vinculación de la comunidad mediante campañas publicitarias y la activa participación en procesos de políticas públicas ambientales y educativas, para aportar al replanteamiento e implementación según cada contexto.

La investigación sobre la educación y la cultura hídricas es un aspecto que se encuentra en un estado inicial y emergente, generado por la crisis ambiental y el detrimento hídrico, lo cual requiere análisis multidisciplinarios e intersectoriales, el aporte de diversos actores sociales y el trabajo conjunto y constante entre profesionales e integrantes de la comunidad.

Desde esta óptica, se concibe que para trazar un cambio en el actuar del individuo frente al entorno natural, es necesario identificar la responsabilidad de todos los actores que influyen en el estado actual de este recurso vital, así como el papel de las industrias, los sectores pecuarios, el sector agrícola y demás actividades económicas que afectan en mayor proporción su calidad y disponibilidad.

\section{Referencias}

Angarita, R. D., Duarte, J. E. y Fernández, F. H. (2018). Desarrollo de un MEC para la creación de cultura ciudadana sobre el uso del recurso hídrico en estudiantes de educación básica. Revista Espacios, 39(15). 1-15. Recuperado de http://www.revistaespacios.com/a18v39n15/ a18v39n15p19.pdf

Bolaños, J. D. B. (2017). Gestor integral del recurso hídrico. Un experto necesario ante la vulnerabilidad socionatural. InterSedes, 18(38). Recuperado de https://revistas.ucr.ac.cr/index. php/intersedes/article/view/32672

Bonilla, G.A. y Vera, B. (2011). ¿Cómo influye la educación ambiental en la cultura? Escritos sobre la biología y su enseñanza. 4(6). 173-181. Recuperado de https:/www.researchgate.net/ publication/304551239_Como_influye_la_educacion_ambiental_en_la_cultura

Campos, M. C. y García Rivera, G. (2017). Aproximación a la ecocrítica y la ecoliteratura: literatura juvenil clásica e imaginarios del agua. Revista de estudios sobre lectura, 16(2), 95-106. Recuperado de https://revista.uclm.es/index.php/ocnos/article/view/ocnos_2017.16.2.1511 
Carrillo, C. V., Carrillo, M. y Pena, M. (2017). Poéticas del agua. Entre la experiencia estética y la conciencia ambiental. Revista Iberoamericana de Ciencia, Tecnología y Sociedad-CTS, 12(35), 243-259. Recuperado de http://www.revistacts.net/volumen-12-numero-35/330-dossier/795poeticas-del-agua-entre-la-experiencia-estetica-y-la-conciencia-ambiental

Carrillo, J.D. y Cacua, S.C. (2019). Educación ambiental en Colombia: Hacia un óptimo desarrollo sostenible. Dialéctica. Revista de Investigación Educativa, (19(1). 170-182.http://portal. amelica.org/ameli/jatsRepo/88/88741012/html/

Cárdenas, C., Reyes, J. y Bustos, E. (2017). Perspectivas sobre la educación hídrica para la cuenca baja del río Fucha. Experiencia en la localidad de Fontibón. Escritos sobre la biología y su enseñanza, 10(número extraordinario) 518-524. https://doi.org/10.17227/bio-grafia. extra2017-7145

Cárdenas, C. (2018). Perspectivas comunitarias sobre educación hídrica para la cuenca baja del río Fucha. Experiencia en la localidad de Fontibón. (Tesis de maestría. Universidad Distrital Francisco José de Caldas. Colombia). https://repository.udistrital.edu.co/bitstream/ handle/11349/8885/C\%C3\%A1rdenasHerreraCarolina2018.pdf?sequence $=2 \&$ isAllowed $=y$

Carroll, N. J. (2015). Informal Water Education and Public Outreach. Journal of Contemporary Water Research \& Education, 156(1), 1-4. https://doi.org/10.1111/j.1936-704X.2015.03199.x

Castelltor, A. (2015). Actividades que contribuyen a la promoción de una nueva cultura del agua. Comunicaçóes, 22(2), 363-389. Recuperado de https://www.metodista.br/revistas/revistasunimep/index.php/comunicacoes/article/view/2340

Cazau, P. (2006). Introducción a la investigación en ciencias sociales. (Tercera Edición ed.). Buenos Aires, Argentina. Recuperado de https://alcazaba.unex.es/asg/400758/MATERIALES/ INTRODUCCI\%C3\%93N\%20A\%20LA\%20INVESTIGACI\%C3\%93N\%20EN\%20 CC.SS..pdf

Correa, L., Pascuas, Y. y Marlés, C. (2016). Desafíos para asumir la educación y la cultura ambiental. Revista Horizontes Pedagógicos. 18(34), 43-42. Recuperado de https://horizontespedagogicos. ibero.edu.co/article/view/18103

Correa, L., López, L., \& Vergara, M. (2020). Investigaciones de representaciones sociales sobre fuentes hídricas y condiciones para la preservación hídrica (2013-2019). Revista Espacios. 41(29). 329-344. Recuperado de http://es.revistaespacios.com/a20v41n29/20412924.html

Correa, L. (2020). Representaciones sociales sobre condiciones culturales-educativas para la renaturalización hídrica. Política pública ambiental y actores sociales. (Tesis doctoral. Universidad de la Amazonia. Colombia).

Damonte, G., y Lynch, B. (2016). Cultura, política y ecología política del agua: una presentación. Anthropologica, 34(37), 5-12. Recuperado de http://revistas.pucp.edu.pe/index.php/ anthropologica/article/view/15617

Delgado, S. M., Trujillo, J. M. y Torres, M. A. (2013). La huella hídrica como una estrategia de educación ambiental enfocada a la gestión del recurso hídrico: ejercicio con comunidades rurales de Villavicencio. Revista Luna Azul, (36), 70-77. Recuperado de https://www.redalyc. org/pdf/3217/321728584006.pdf

Díez, J. (2014). El agua: una herramienta clave en la educación para el desarrollo sostenible. Revista Brasileira de Pesquisa em Educaçáo em Ciências, 14(2), 109-123. Recuperado de https:// periodicos.ufmg.br/index.php/rbpec/article/view/4354

Fernández, J. y Rodríguez, F. (2016). Los procesos de enseñanza-aprendizaje relacionados con el agua en el marco de las hipótesis de transición. Revista Eureka sobre enseñanza y divulgación de las ciencias, 14(1), 227-243. Recuperado de http://ojs.uca.es/index.php/tavira/article/view/882

Figueroa, A. (2017). Cultura ambiental para mitigar la contaminación de las microcuencas proveedoras de agua a la ciudad de Loja. INNOVA Research Journal, 2(10.1), 25-35. https://doi. org/10.33890/innova.v2.n10.1.2017.472 
Frausto, J. (2015). Gestión y cultura del agua en Nuevo Laredo, Tamaulipas. Frontera norte, 27(53), 89-114. Recuperado de http://www.scielo.org.mx/scielo.php?pid=S0187$73722015000100004 \&$ script=sci_arttext

Freitas, N. y Marín, F. (2015). Educação ambiental e água: concepçóes e práticas educativas em escolas municipais. Nuances: estudos sobre Educação, 26(1). 234-253. Recuperado de http:// revista.fct.unesp.br/index.php/Nuances/article/view/2813

Fuentes, A. (2012). Movimiento social por una nueva cultura del agua en España. Espacios Públicos, 15(35), 96-113. Recuperado de https://www.redalyc.org/pdf/676/67624803007.pdf

Galván, L., Ouariachi, T., Pozo, M. y Gutiérrez, J. (2018). Outstanding videogames on water: A quality assessment review based on evidence of narrative, gameplay and educational criteria. Water, 10(10), 1404. Recuperado de https://www.mdpi.com/2073-4441/10/10/1404

Gil, B., Quílez, M., Peńa, M., Hervás, A. y Muñoz, A. (2018). Los niños y las niñas de infantil piensan, actúan y hablan sobre el comportamiento del aire y del agua. Revista de investigación y experiencias didácticas, 36(1), 163-180. Recuperado de https://www.raco.cat/index.php/ Ensenanza/article/view/335279

González, M., y Arzaluz, M. (2011). El programa de cultura del agua en el noreste de México. ¿Concepto utilitario, herramienta sustentable o requisito administrativo? Región y sociedad, 23(51), 123-160. Recuperado de http://www.scielo.org.mx/scielo.php?script=sci_arttext\&pi $\mathrm{d}=\mathrm{S} 1870-39252011000200005$

González, F. y Vega, S. (2018). Cultura hídrica. Agua y cultura en la región Mazahua. Revista Luna Azul, (46), 258-284. Recuperado de https://www.redalyc.org/jatsRepo/3217/321759619014/321759619014.pdf

Grigg, N. S. (2019). IWRM and the Nexus Approach: Versatile Concepts for Water Resources Education. Journal of Contemporary Water Research \& Education, 166(1), 24-34. Recuperado de https://onlinelibrary.wiley.com/doi/full/10.1111/j.1936-704X.2019.03299.x

Haynes, S. y Cech, T. (2015). One World One Water Center for Urban Water Education and Stewardship. Journal of Contemporary Water Research \& Education, 156(1), 37-47. Recuperado de https://onlinelibrary.wiley.com/doi/full/10.1111/j.1936-704X.2015.03202.x

Hernández, M. y Gómez, G. (2014). Actitudes ambientales hacia el agua, una exploración en estudiantes del municipio de Ventaquemada (Boyacá). Revista Luna Azul, (39), 40-62. Recuperado de http://www.scielo.org.co/scielo.php?script=sci_arttext\&pid=S1909-24742014000200004

Hernández, S. y Palacios, S. (2016). Importancia del componente social en el manejo del recurso hídrico, río El Encano, humedal Ramsar, La Cocha (Nariño, Colombia). Revista Luna Azul, (42), 200-216. Recuperado de https://www.redalyc.org/pdf/3217/321744162008.pdf

Moreno, J., Vahos, S. y Mazo, C. (2019) Videojuego para la enseñanza del cuidado del agua. Tecnológicas 22(45), 59-72, 2019. Recuperado de https://doi.org/10.22430/22565337.1091

Jácome, S. Ordóńez, A., Cerón, G. y Villaquirán, A. (2018). Mapeo sistemático del uso de las tecnologías de la información y la comunicación en la diabetes tipo 2. Revista Cubana de Información en Ciencias de la Salud, 29(4), 1-14. Recuperado de http://scielo.sld.cu/pdf/ics/ v29n4/a05_1226.pdf

Jardioui, Y., Elachqar, A., Kaddari, F. y Ijjaalib, M. (2015). Education: Essential Approach For A Better Water Management. Procedia-Social and Behavioral Sciences, 191, 896-899. Recuperado de https://www.sciencedirect.com/science/article/pii/S1877042815026890

Koberwein, A. (2017). Transformaciones sociales y conocimiento en un contexto de crisis hídrica en la provincia de Córdoba, Argentina. Anthropologica, 35(38), 95-118.

Kulcsar, L., Aistrup, J., Bulatewicz, T., Peterson, J., Welch, S. y Steward, D. (2016). Water and society: Interdisciplinary education in natural resources. Journal of Contemporary Water Research \& Education. 158(1), 120-131.

Lara, A. y Otaño, L.C. (2017). Agua, cultura y educación ambiental. XXVI Congreso Nacional de agua (Conagua). 
López, M. (2017). Programas de cultura del agua en Nuevo León. Análisis de los efectos de las campańas publicitarias en la disminución del consumo en la población. Trayectorias. Revista de ciencias sociales de la Universidad Autónoma de Nuevo León, 19(45), 101-126.

Macpherson, C., y Macpherson, L. A. (2017). Culture and the commodification of water in Samoa. Asia Pacific Viewpoint, 58(1), 86-98.

Mahler, R. (2019). University Science-Based Education Positively Impacts College Student Views of Water Issues. Natural Sciences Education, 48(1).

Marlés, C., Peña, P. y Gómez, C. (2017). La lúdica como estrategia para la educación y cultura ambiental en el contexto universitario. Revista UNIMAR, 35(2), 283 -292. Recuperado de: http://editorial.umariana.edu.co/revistas/index.php/unimar/article/view/1540/pdf

Marlés, C., Rojas, G., \& Correa, L. (2020). Actitudes ambientales hacia la gestión hídrica: estudio de percepción en la Universidad de la Amazonia, Colombia. Revista Espacios. 41(35). 213-228. Recuperado de: http://revistaespacios.com/a20v41n35/a20v41n35p17.pdf

Mazabel, D., y Fragoso, A. (2012). Diversidad cultural y gestión del agua. Lecciones desde una región mazahua del Estado de México. Antropología Experimental, 30(12).395-408

Missingham, B. (2013). Participatory learning and popular education strategies for water education. Journal of Contemporary Water Research \& Education, 150(1), 34-40.

Miranda, L. (2013). Cultura ambiental: un estudio desde las dimensiones de valor, creencias, actitudes y comportamientos ambientales. Producción + limpia. 8(2). 94-105. Recuperado de https://dialnet.unirioja.es/servlet/articulo?codigo=5012134

Monja, P.C. (2018). El cuento infantil como herramienta educativa para el ahorro del agua potable a causa de su uso irracional (Tesis de pregrado. Universidad San Ignacio de Loyola, Perú). http://repositorio.usil.edu.pe/bitstream/USIL/8885/1/2018_Monja-Beltran.pdf

Moreira, C., Araya, F., y Charpentier, C. (2015). El agua como parte de la cultura de las comunidades rurales: un análisis para la cuenca del río San Carlos. Revista Tecnología en Marcha, 28(2), 126-140. Recuperado de https:/www.scielo.sa.cr/pdf/tem/v28n2/0379-3982tem-28-02-00126.pdf

Ortega, J. (2015). Gestión y cultura del agua en Nuevo Laredo, Tamaulipas. Frontera norte, 27(53), 89-114. Recuperado de http://www.scielo.org.mx/scielo.php?script=sci_arttext\&pid $=$ S0187-73722015000100004

Ortega, D., y Peńa, A. (2016). Análisis crítico de las campañas de comunicación para fomentar la cultura del agua en México. En Comunicación y sociedad, 1(26), 223-246. Recuperado de http://www.scielo.org.mx/scielo.php?script=sci_arttext\&pid=S0188-252X2016000200223

Oyaga, R., Calderón, J., Olaya, N., Enamorado, J, y Atencio, F. (2017). Formas dialógicas inclusivas en educación ambiental para la democratización social de la cultura del agua. Revista Espacios. 38(30). 1-26. Recuperado de https://revistaespacios.com/a17v38n30/a17v38n30p26.pdf

Panéz, A. (2017). Agua. Territorio en América Latina. Contribuciones a partir del análisis de estudios sobre conflictos hídricos en Chile. Revista Rupturas, 8(1), 193-217. Recuperado de https://www.scielo.sa.cr/pdf/rup/v8n1/2215-2989-rup-8-01-193.pdf

Pérez, R. (2015). La nueva cultura del agua, el camino hacia una gestión sostenible. Cuadernos de Trabajo Hegoa, (68), 1-53. Recuperado de: https://ojs.ehu.eus/index.php/hegoa/article/ view/15672

Read, L., y García, M. (2015). Water diplomacy: Perspectives from a group of interdisciplinary graduate students. Journal of Contemporary Water Research \& Education, 155(1), 11-18. DOI: 10.1111/j.1936-704X.2015.03191.x

Sammel, A. (2016). Her beauty and her terror: A case study exploring the framing of water and extreme water events within formal education in Queensland, Australia and Saskatchewan, Canada. Geoforum, 76(1). 164-175. 
Sanz, B. (2018). El agua en el hogar, un proyecto para educación primaria. (Tesis de pregrado. Universidad de Valladolid). España.

Severiche, C., Gómez, E. y Morales, J. (2016). La educación ambiental como base cultural y estrategia para el desarrollo sostenible. Telos. Revista de estudios interdisciplinarios en Ciencias Sociales. 18(2). 266-281. Recuperado de https://www.redalyc.org/pdf/993/99345727007.pdf

Sierra, W., Medina, I. y Aguilera, H. (2018). Ambientalización del currículo en educación superior y consumo de agua en los hogares de estudiantes universitarios. Gestión y Ambiente, 21(2), 263-275.

Topete, N., Verdín, E., y Galaviz, I. (2019). Analfabetismo hídrico en el municipio de Xalisco, Nayarit. El reto para mejorar la gobernanza del agua y la seguridad hídrica. RICSH Revista Iberoamericana de las Ciencias Sociales y Humanísticas, 8(16), 111-138. Recuperado de http:// www.ricsh.org.mx/index.php/RICSH/article/view/177

Unesco (2018). Informe de actividades relacionadas al PHI. Programas y grupos de trabajo del PHI http://www.unesco.org/new/fileadmin/MULTIMEDIA/FIELD/Montevideo/pdf/IHPEducyCdelAgua-.pdf

Unesco (2020a). La seguridad hídrica y los objetivos de desarrollo sostenible. Manual de capacitación para tomadores de decisión. Documento técnico No.42. https://lawetnet.org/wp-content/ uploads/2021/02/Manual-La-Seguridad-Hidrica-y-los-ODS.pdf

Unesco (2020b). Informe Mundial de las Naciones Unidas sobre el Desarrollo de los Recursos Hídricos 2020: Agua y Cambio Climático, París, Unesco. Recuperado dehttps://unesdoc. unesco.org/ark:/48223/pf0000373611.locale=es

Velásquez, J. D. (2014). Una guía corta para escribir Revisiones Sistemáticas de Literatura Parte 2. Dyna, 81(188), 9-10. https://revistas.unal.edu.co/index.php/dyna/article/view/46758

Venckute, M., Moreira, M., \& Figueiredo, M. (2017). La educación como instrumento para reducción de la pegada hídrica de los jóvenes. Millenium, 2(4), 101-111. https://www. researchgate.net/publication/322069419_Education_as_a_tool_to_reduce_the_water_footprint_of_young_people

Venegas, M. Moncada, A., y Alvis, R. (2016). Cocreación como metodología para la apropiación social de la ciencia y la tecnología (ASCYT) del recurso agua. Caso Urabá-Antioqueño, Colombia. El Ágora USB, 16(1), 277-286. Recuperado de https://www.redalyc.org/ pdf/4077/407755353014.pdf

Verdín, E., Topete, N., Galaviz, I., Ponce, A., y Soriano, E. (2016). Legislación y la cultura del agua: análisis correlacional en Nayarit. Revista Iberoamericana de las Ciencias Biológicas y Agropecuarias: CIBA, 5(9), 115. Recuperado de https://dialnet.unirioja.es/servlet/ articulo?codigo $=5662200$ 